\title{
V. RESULTS OF SHORE LABORATORY STUDIES ON TERTIARY AND QUATERNARY FORAMINIFERA FROM LEG 26
}

\author{
W. H. Akers, Chevron Oil Company, New Orleans, Louisiana
}

Tables 1 through 15 show the occurrence of Tertiary and Quaternary planktonic foraminifera in samples studied from cores taken on Leg 26. The symbols used in the tables are as follows.

0 - Absent

1 - Rare (1-10 specimens)
2 - Moderately rare (11-25 specimens)

3 - Common (26-50 specimens)

4 - Abundant (51-100 specimens)

5 - Very abundant $(>100$ specimens $)$

$\mathrm{R}$ - Reworked

TABLE 1

Distribution of Foraminifera, Site $\mathbf{2 5 0}$

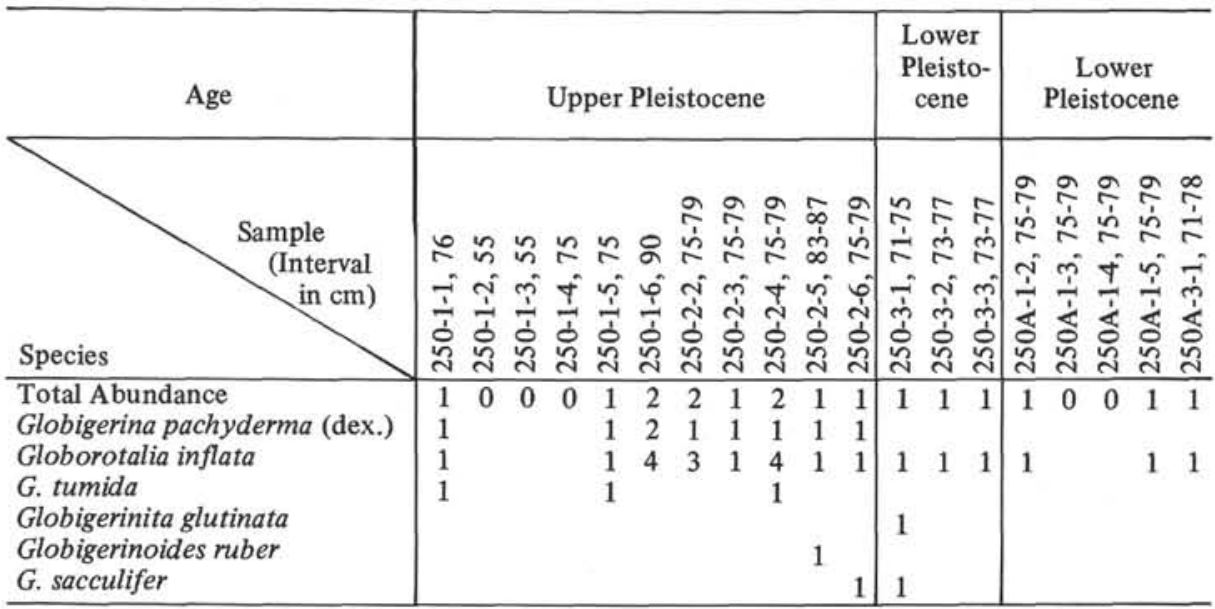

TABLE 2

Distribution of Foraminifera, Site 250 - Continued

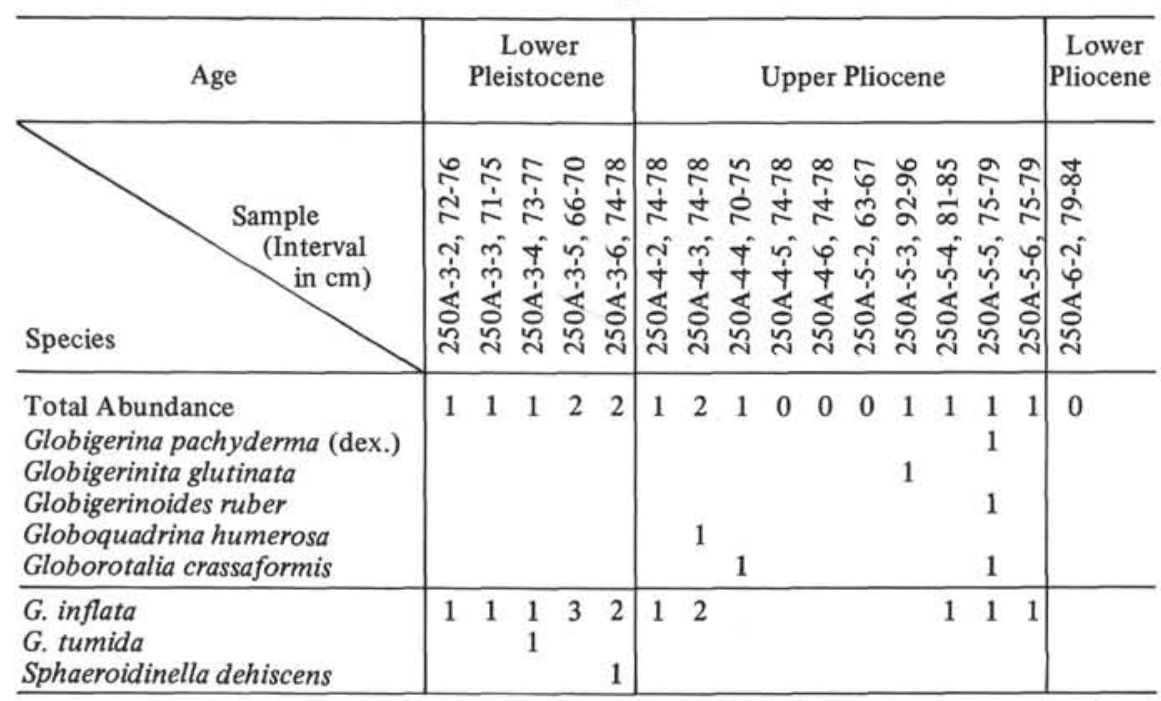




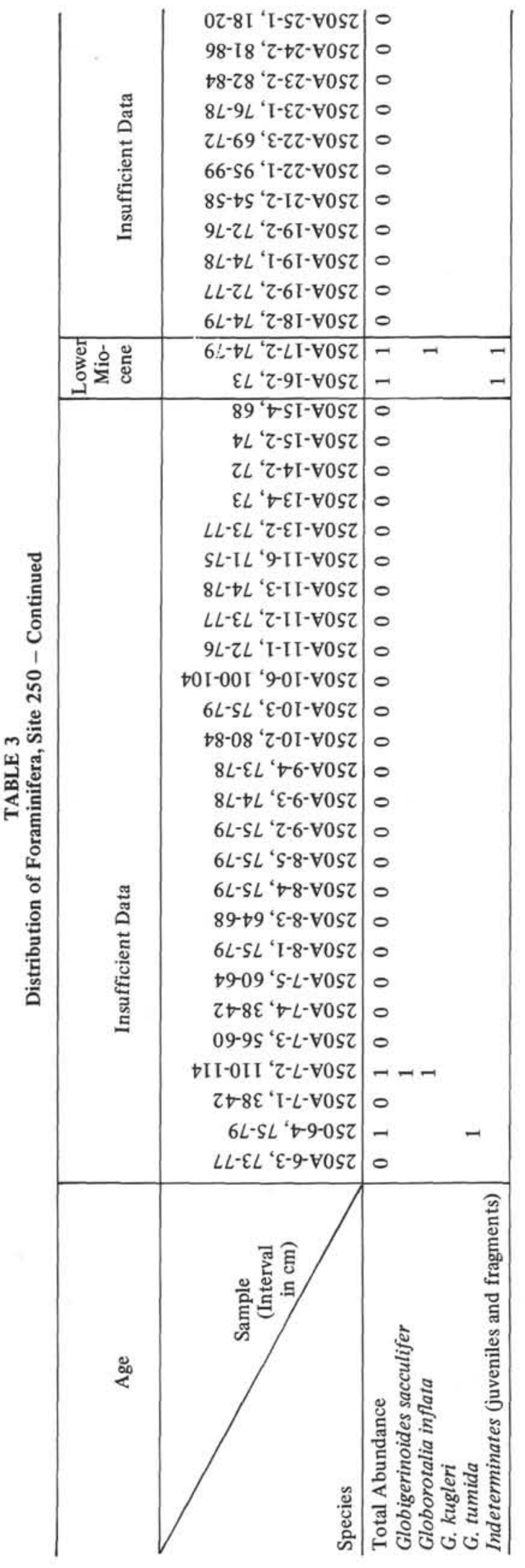

TABLE 4

Distribution of Foraminifera, Site 251

\begin{tabular}{|c|c|c|c|c|c|c|c|}
\hline Age & \multicolumn{7}{|c|}{ Quaternary } \\
\hline $\begin{array}{r}\text { Sample } \\
\text { (Interval } \\
\text { in } \mathrm{cm})\end{array}$ & 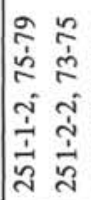 & 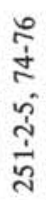 & 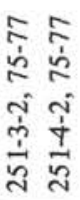 & 5 & $\hat{s}$ & & \\
\hline $\begin{array}{l}\text { Total Abundance } \\
\text { Globigerina bulloides } \\
\text { G. pachyderma (dex.) } \\
\text { Globigerinita glutinata } \\
\text { Globigerinoides conglobatus } \\
\text { G. ruber }\end{array}$ & $\begin{array}{ll}5 & 5 \\
4 & 5 \\
3 & 5 \\
1 & 1 \\
1 & 1\end{array}$ & $\begin{array}{l}5 \\
5 \\
5 \\
2 \\
1 \\
2\end{array}$ & $\begin{array}{ll}4 & 3 \\
5 & 1 \\
5 & 5 \\
& 3 \\
& \\
1 & \end{array}$ & $\begin{array}{l}5 \\
4 \\
5 \\
2\end{array}$ & $\begin{array}{l}5 \\
5 \\
4\end{array}$ & $\begin{array}{l}5 \\
5 \\
5 \\
5\end{array}$ & $\begin{array}{l}5 \\
5 \\
5\end{array}$ \\
\hline $\begin{array}{l}\text { G. sacculifer } \\
\text { Globoquadrina dutertrei } \\
\text { G. humerosa } \\
\text { Globorotalia crassaformis } \\
\text { G. cultrata (sin.) }\end{array}$ & $\begin{array}{ll}1 & \\
1 & \\
1 & 4\end{array}$ & $\begin{array}{l}1 \\
1 \\
4 \\
1\end{array}$ & $\begin{array}{l}1 \\
1\end{array}$ & 1 & $\begin{array}{l}3 \\
1\end{array}$ & $\begin{array}{l}1 \\
1\end{array}$ & 4 \\
\hline $\begin{array}{l}\text { G. hirsuta } \\
\text { G. inflata } \\
\text { G. scitula } \\
\text { G. tosaensis } \\
\text { G. truncatulinoides }\end{array}$ & $\begin{array}{ll}2 & \\
5 & 5 \\
1 & \\
1 & 1 \\
5 & 4\end{array}$ & $\begin{array}{l}1 \\
5 \\
1 \\
1 \\
4\end{array}$ & $\begin{array}{ll}5 & \\
1 & 1 \\
1 & \\
\end{array}$ & $\begin{array}{l}5 \\
1 \\
1 \\
1\end{array}$ & $\begin{array}{l}5 \\
1 \\
2\end{array}$ & $\begin{array}{l}5 \\
1 \\
2\end{array}$ & 5 \\
\hline $\begin{array}{l}\text { G. tumida } \\
\text { Hastigerina siphonifera } \\
\text { Orbulina universa } \\
\text { Pulleniatina obliquiloculata } \\
\text { Sphaeroidinella dehiscens }\end{array}$ & $\begin{array}{ll}1 & \\
2 & 1 \\
4 & 3\end{array}$ & $\begin{array}{l}1 \\
4 \\
1\end{array}$ & $\begin{array}{l}1 \\
1\end{array}$ & 4 & $\begin{array}{l}3 \\
1\end{array}$ & $\begin{array}{l}1 \\
5 \\
1\end{array}$ & \\
\hline
\end{tabular}


TABLE 5

Distribution of Foraminifera, Site 251 - Continued

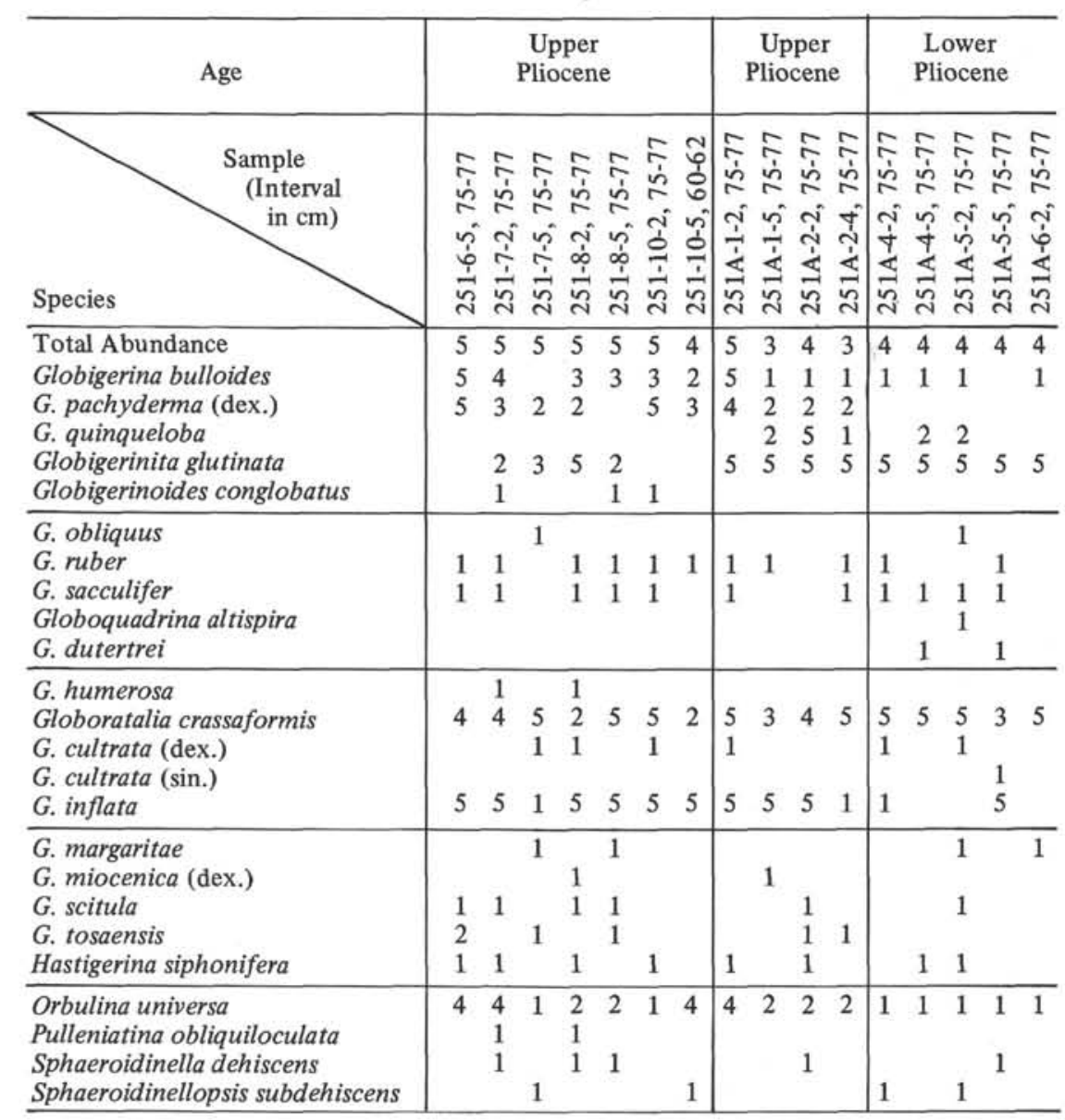


TABLE 6

Distribution of Foraminifera, Site 251 - Continued

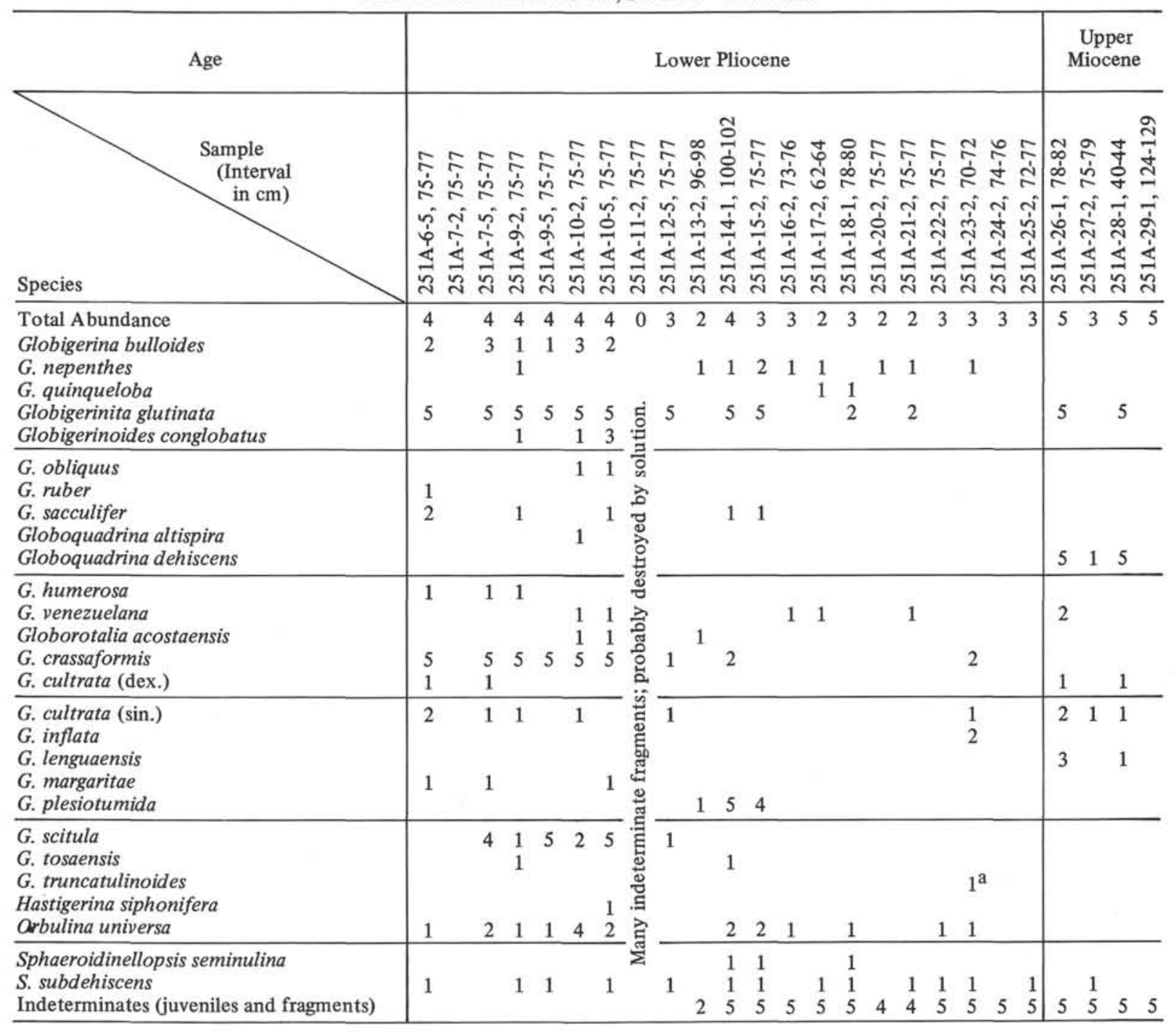

TABLE 7

Distribution of Foraminifera, Site 252

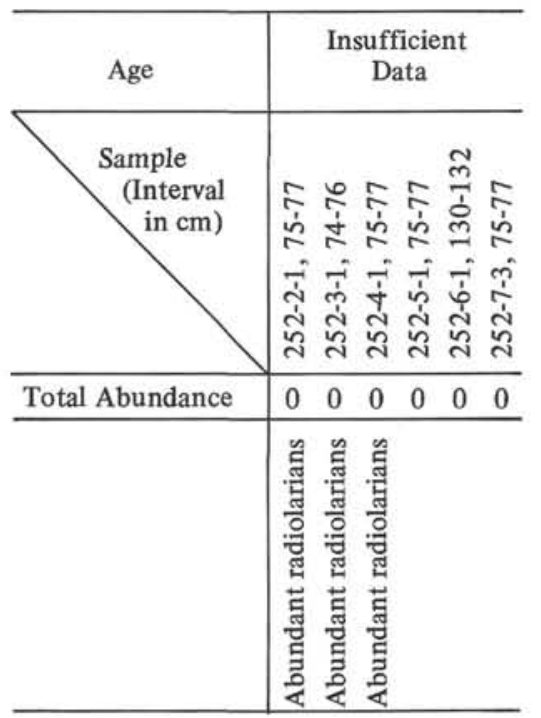


TABLE 8

Distribution of Foraminifera, Site 253

\begin{tabular}{|c|c|c|c|c|c|c|c|c|c|c|c|c|c|c|}
\hline Age & $\begin{array}{c}\text { Qua- } \\
\text { ter- } \\
\text { nary }\end{array}$ & & & Plioc & cene & & & & & & & & & \\
\hline$\underbrace{\substack{\text { Sample } \\
\text { (Interval } \\
\text { ind }}}_{\text {in } \mathrm{cm} \text { ) }}$ & 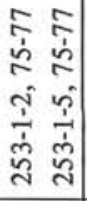 & 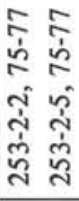 & $\begin{array}{l}\hat{\sim} \\
\tilde{r} \\
\tilde{n} \\
\tilde{n} \\
\tilde{n}\end{array}$ & 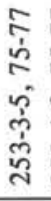 & 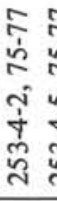 & 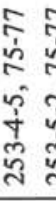 & 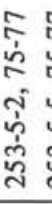 & 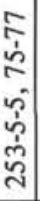 & 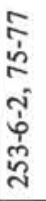 & 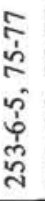 & 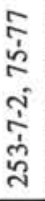 & 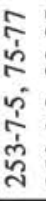 & & 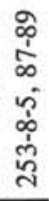 \\
\hline $\begin{array}{l}\text { Total Abundance } \\
\text { Candeina nitida } \\
\text { Globigerina bulloides } \\
\text { G. conglomerata } \\
\text { G. nepenthes } \\
\text { G. quinqueloba }\end{array}$ & $\begin{array}{ll}5 & 5 \\
1 & \\
& 1\end{array}$ & $\begin{array}{ll}5 & 5 \\
1 & 1 \\
1 & 1\end{array}$ & $\begin{array}{l}5 \\
1 \\
1\end{array}$ & $\begin{array}{l}5 \\
2 \\
1\end{array}$ & $\begin{array}{l}5 \\
1 \\
1\end{array}$ & $\begin{array}{ll}5 & 5 \\
1 & 2 \\
& 5 \\
& 3\end{array}$ & $\begin{array}{l}5 \\
2 \\
5 \\
3\end{array}$ & \begin{tabular}{l|l}
5 \\
1
\end{tabular} & $\begin{array}{l}3 \\
5\end{array}$ & $\begin{array}{l}5 \\
1 \\
2 \\
5\end{array}$ & 5 & 5 & 5 & 5 \\
\hline $\begin{array}{l}\text { Globigerinita glutinata } \\
\text { Globigerinoides conglobatus } \\
\text { G. mitra } \\
\text { G. obliquus } \\
\text { G. ruber } \\
\end{array}$ & \begin{tabular}{ll|}
5 & 5 \\
4 & 5 \\
& \\
5 & 5
\end{tabular} & $\begin{array}{ll}4 & 5 \\
5 & \end{array}$ & $\begin{array}{l}2 \\
5\end{array}$ & $\begin{array}{l}1 \\
5\end{array}$ & $\begin{array}{l}1 \\
5\end{array}$ & 5 & & 2 & $\begin{array}{l}4 \\
1\end{array}$ & $\begin{array}{l}4 \\
1 \\
5\end{array}$ & $\begin{array}{l}1 \\
4\end{array}$ & 5 & 2 & 1 \\
\hline $\begin{array}{l}\text { G. sacculifer } \\
\text { G. sacculifer fistulosus } \\
\text { Globoquadrina altispira } \\
\text { G. dehiscens } \\
\text { G. dutertrei } \\
\end{array}$ & $\begin{array}{ll}1 & 1\end{array}$ & $\begin{array}{ll}5 & 5 \\
& 2 \\
4 & 4\end{array}$ & $\begin{array}{l}5 \\
1 \\
2\end{array}$ & $\begin{array}{l}5 \\
1\end{array}$ & & $\begin{array}{ll}5 & 5 \\
1 & 2\end{array}$ & $\begin{array}{l}5 \\
2\end{array}$ & 5 & $\begin{array}{l}4 \\
1 \\
5\end{array}$ & $\begin{array}{l}5 \\
1 \\
5\end{array}$ & $\begin{array}{l}1 \\
5\end{array}$ & $\begin{array}{l}1 \\
5\end{array}$ & $\begin{array}{l}4 \\
3 \\
5\end{array}$ & $\begin{array}{l}5 \\
3 \\
5\end{array}$ \\
\hline $\begin{array}{l}\text { G. venezeulana } \\
\text { Globorotalia crassaformis } \\
\text { G. cultrata (dex.) } \\
\text { G. cultrata }(\text { sin.) } \\
\text { G. inflata } \\
\end{array}$ & $\begin{array}{ll}3 & 5 \\
1^{\mathrm{a}} & \\
2 & 2 \\
1 & \end{array}$ & $\begin{array}{ll}1 & 1 \\
5 & 3 \\
3 & 5\end{array}$ & $\begin{array}{l}1 \\
5\end{array}$ & $\begin{array}{l}5 \\
1\end{array}$ & 3 & 35 & $\begin{array}{l}1 \\
5\end{array}$ & $\begin{array}{l}1 \\
5\end{array}$ & 1 & $\begin{array}{l}1 \\
4 \\
4\end{array}$ & 1 & 1 & $\begin{array}{l}1 \\
1\end{array}$ & $\begin{array}{l}1 \\
1\end{array}$ \\
\hline $\begin{array}{l}\text { G. lenguaensis } \\
\text { G. margaritae } \\
\text { G. miocenica (dex.) } \\
\text { G. multicamerata } \\
\text { G. plesiotumida } \\
\end{array}$ & $1^{\mathrm{a}}$ & 14 & $\begin{array}{l}5 \\
1\end{array}$ & $\begin{array}{l}5 \\
1\end{array}$ & $\begin{array}{l}5 \\
1\end{array}$ & 1 & & 1 & 1 & & 1 & 2 & $\begin{array}{l}2 \\
1\end{array}$ & $\begin{array}{l}5 \\
1\end{array}$ \\
\hline $\begin{array}{l}\text { G. scitula } \\
\text { G. truncatulinoides } \\
\text { G. new species } \\
\text { Hastigerina siphonifera } \\
\text { Orbulina universa }\end{array}$ & \begin{tabular}{ll|}
2 & 2 \\
5 & 1 \\
4 & 1 \\
5 & 5
\end{tabular} & $\begin{array}{ll} & 2 \\
1 & 1 \\
5 & 4\end{array}$ & $\begin{array}{l}1 \\
5\end{array}$ & $\begin{array}{l}1 \\
5\end{array}$ & $\begin{array}{l}1 \\
5\end{array}$ & & 5 & $\begin{array}{l}1 \\
4\end{array}$ & $\begin{array}{l}1 \\
4\end{array}$ & $\begin{array}{l}1 \\
5\end{array}$ & 1 & 3 & 4 & 5 \\
\hline $\begin{array}{l}\text { Pulleniatina obliquiloculata } \\
\text { Sphaeroidinella dehiscens } \\
\text { Sphaeroidinellopsis kochi } \\
\text { S. seminulina } \\
\text { S. subdehiscens } \\
\text { Indeterminates (juveniles and fragments) }\end{array}$ & $\begin{array}{ll}1 & 1 \\
1 & 1\end{array}$ & $\begin{array}{ll}3 & \\
2 & 1 \\
1 & 2\end{array}$ & $\begin{array}{l}3 \\
3 \\
1\end{array}$ & $\begin{array}{l}3 \\
3\end{array}$ & $\begin{array}{l}1 \\
3 \\
1\end{array}$ & 4 & $\begin{array}{l}1 \\
5 \\
1\end{array}$ & $\begin{array}{l}1 \\
5 \\
1\end{array}$ & $\begin{array}{l}1 \\
5 \\
1\end{array}$ & $\begin{array}{l}1 \\
5\end{array}$ & $\begin{array}{l}4 \\
5\end{array}$ & $\begin{array}{l}2 \\
5\end{array}$ & $\begin{array}{l}1 \\
5\end{array}$ & 1 \\
\hline
\end{tabular}

${ }^{\mathrm{a}}$ Reworked. 
TABLE 9

Distribution of Foraminifera, Site 253 - Continued

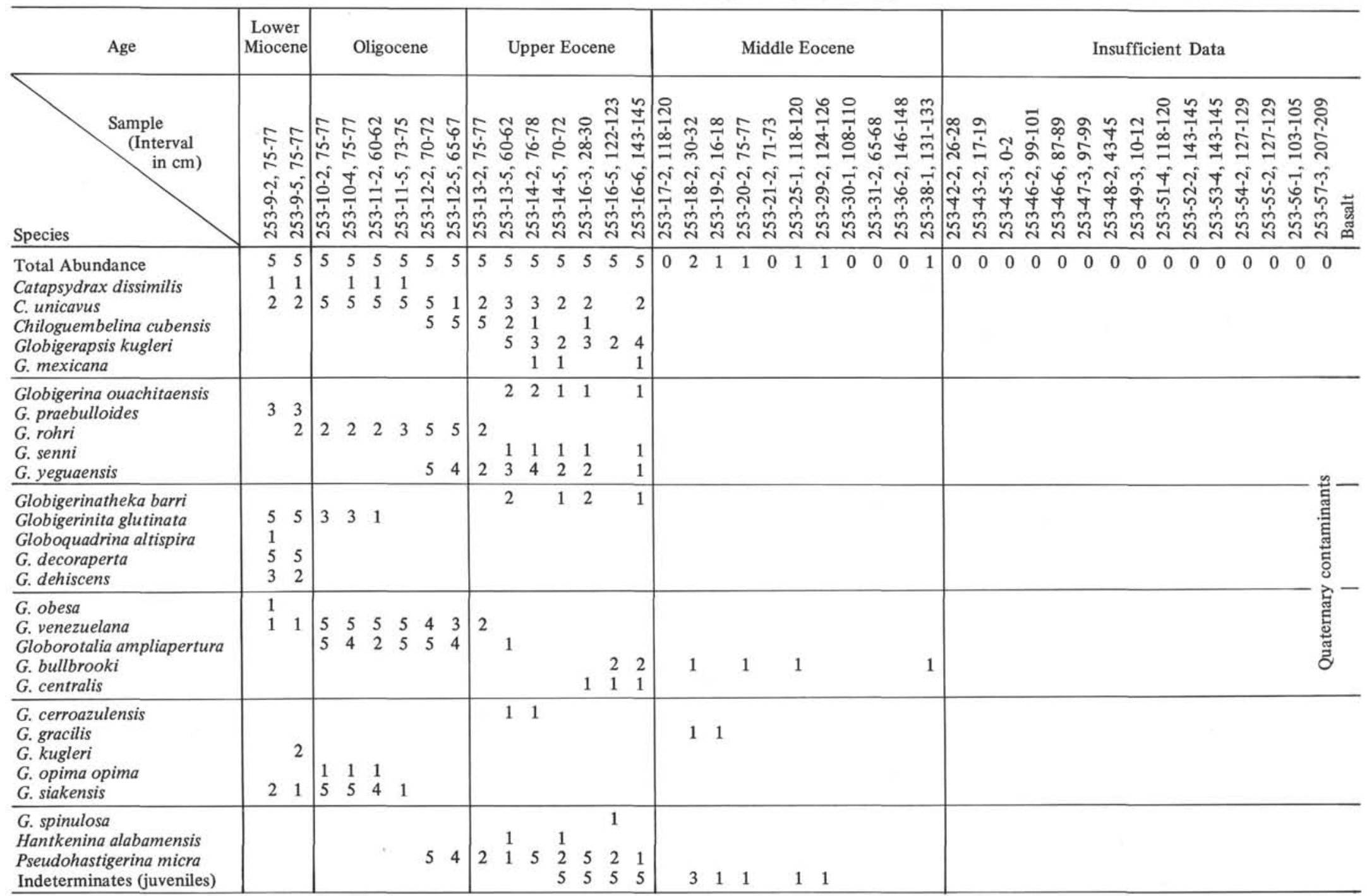


TABLE 10

Distribution of Foraminifera, Site 254

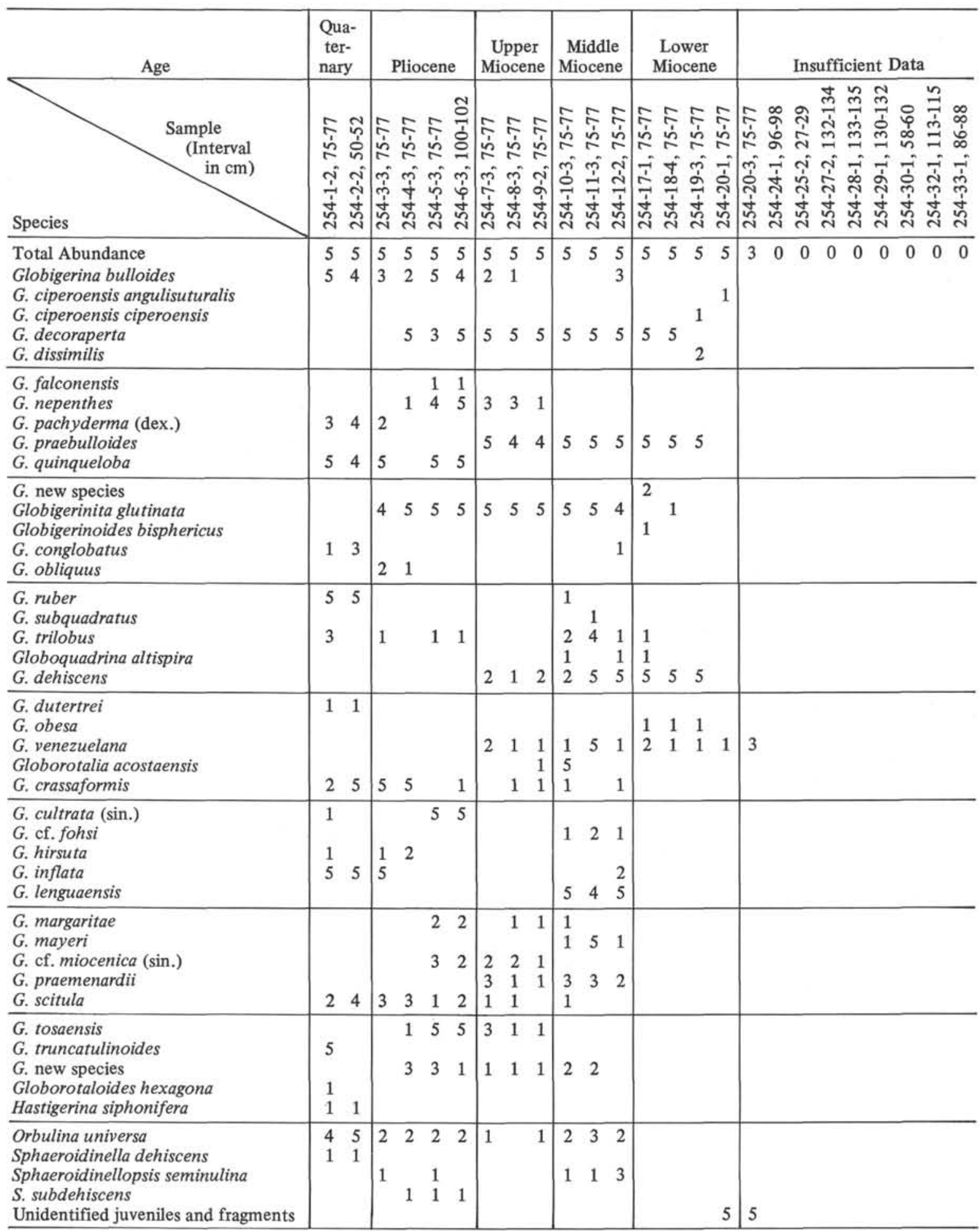


TABLE 11

Distribution of Foraminifera, Site 255

\begin{tabular}{|c|c|c|c|c|c|}
\hline Age ?? & Qu & & Plio. & & $\begin{array}{c}\text { L. } \\
\text { Mio. }\end{array}$ \\
\hline$\underbrace{\begin{array}{c}\text { Sample } \\
\text { (Interval } \\
\text { in } \mathrm{cm})\end{array}}$ & 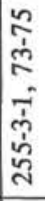 & 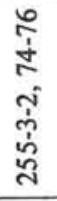 & 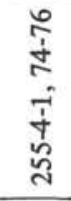 & 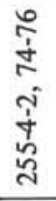 & 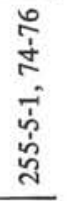 \\
\hline $\begin{array}{l}\text { Total Abundance } \\
\text { Globigerina bulloides } \\
\text { G. decoraperta } \\
\text { G. nepenthes } \\
\text { G. praebulloides } \\
\text { G. prasaepis } \\
\end{array}$ & $\begin{array}{l}5 \\
5 \\
5 \\
4\end{array}$ & $\begin{array}{l}5 \\
3 \\
5 \\
3\end{array}$ & $\begin{array}{l}5 \\
5\end{array}$ & 5 & $\begin{array}{l}5 \\
2 \\
1\end{array}$ \\
\hline $\begin{array}{l}\text { Globigerinita glutinata } \\
\text { Globigerinoides conglobatus } \\
\text { G. ruber } \\
\text { G. trilobus } \\
\text { Globoquadrina altispira } \\
\end{array}$ & $\begin{array}{l}1 \\
2 \\
2\end{array}$ & $\begin{array}{l}1 \\
2 \\
1 \\
1^{\mathrm{a}}\end{array}$ & 2 & 5 & 2 \\
\hline $\begin{array}{l}\text { G. dehiscens } \\
\text { G. humerosa } \\
\text { G. venezuelana } \\
\text { Globorotalia acostaensis } \\
\text { G. ampliapertura } \\
\end{array}$ & 1 & & & $\begin{array}{l}5 \\
2\end{array}$ & $\begin{array}{l}5 \\
1 \\
1 \\
1 \\
1 \\
\end{array}$ \\
\hline $\begin{array}{l}\text { G. crassaformis } \\
\text { G. cultrata ( } \sin .) \\
\text { G. cf. fohsi } \\
\text { G. inflata } \\
\text { G. lenguaensis }\end{array}$ & $\begin{array}{l}5 \\
2 \\
5\end{array}$ & $\begin{array}{l}5 \\
5 \\
5\end{array}$ & 1 & $\begin{array}{l}5 \\
1 \\
5\end{array}$ & \\
\hline $\begin{array}{l}\text { G. margaritae } \\
\text { G. opima opima } \\
\text { G. scitula } \\
\text { G. tosaensis } \\
\text { G. truncatulinoides }\end{array}$ & $\begin{array}{l}1 \\
5\end{array}$ & $\begin{array}{l}3 \\
1\end{array}$ & & 2 & 1 \\
\hline $\begin{array}{l}\text { Orbulina universa } \\
\text { Sphaeroidinella dehiscens } \\
\text { Sphaeroidinellopsis seminulina } \\
\text { S. subdehiscens } \\
\text { Unidentified juveniles and fragments }\end{array}$ & \begin{tabular}{|l}
3 \\
3 \\
1 \\
1
\end{tabular} & $\begin{array}{l}2 \\
1 \\
2^{\mathrm{a}} \\
1\end{array}$ & 5 & 1 & \\
\hline
\end{tabular}

${ }^{\mathrm{a}}$ Reworked

Table 12

Distribution of Foraminifera, Site 256

\begin{tabular}{|c|c|}
\hline Age ?? & Insufficient Data \\
\hline $\begin{array}{l}\text { Imple } \\
\text { (Interval } \\
\text { in } \mathrm{cm} \text { ) }\end{array}$ & 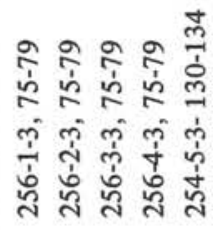 \\
\hline Total Abundance & $\begin{array}{lllll}0 & 0 & 0 & 0 & 0\end{array}$ \\
\hline
\end{tabular}

TABLE 13

Distribution of Foraminifera, Site $\mathbf{2 5 7}$

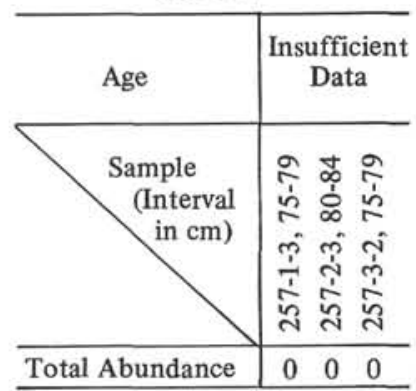


TABLE 14

Distribution of Foraminifera, Site $\mathbf{2 5 8}$

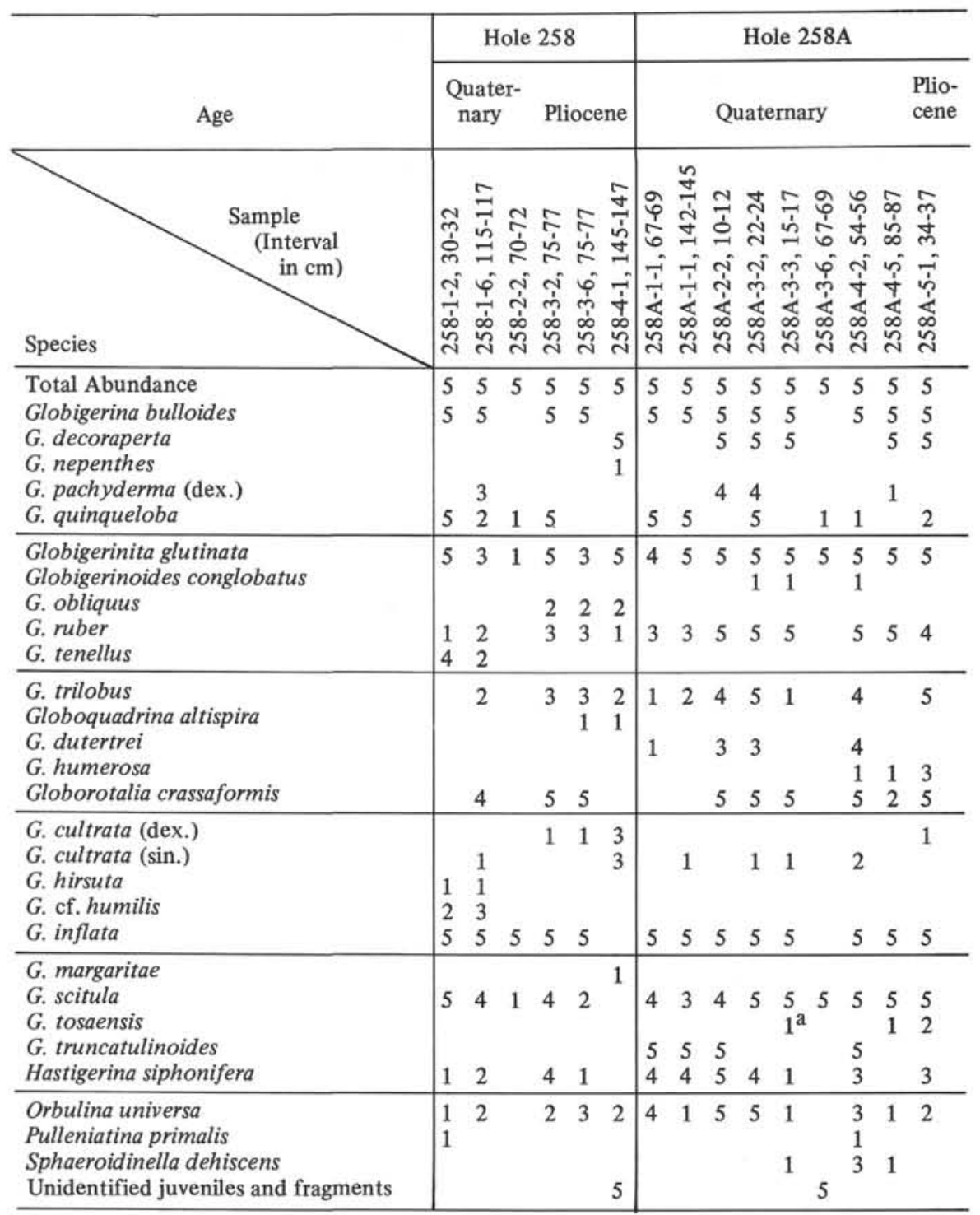

${ }^{a}$ Reworked. 
TABLE 15

Distribution of Foraminifera, Site 258 - Continued

\begin{tabular}{|c|c|c|c|c|c|c|c|c|c|}
\hline \multirow[b]{2}{*}{ Species } & \multicolumn{5}{|c|}{ Pliocene? } & \multicolumn{4}{|c|}{ ?U. Miocene } \\
\hline & $\frac{a}{5}$ & & 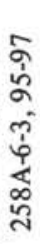 & 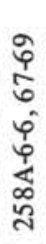 & 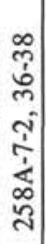 & 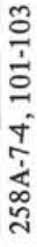 & 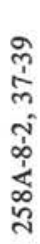 & 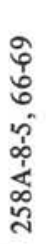 & 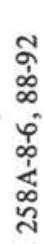 \\
\hline $\begin{array}{l}\text { Total Abundance } \\
\text { Candeina nitida } \\
\text { Globigerina bulloides } \\
\text { G. conglomerata } \\
\text { G. decoraperta } \\
\text { G. nepenthes }\end{array}$ & $\begin{array}{l}5 \\
1 \\
5 \\
5\end{array}$ & $\begin{array}{l}5 \\
2 \\
5 \\
3 \\
5\end{array}$ & $\begin{array}{l}5 \\
5\end{array}$ & $\begin{array}{l}5 \\
1 \\
4\end{array}$ & $\begin{array}{l}4 \\
5\end{array}$ & $\begin{array}{l}5 \\
5 \\
5 \\
1 \\
5 \\
1\end{array}$ & 5 & $\begin{array}{l}5 \\
1 \\
5 \\
5 \\
5 \\
1\end{array}$ & $\begin{array}{l}5 \\
1 \\
5 \\
5 \\
5\end{array}$ \\
\hline $\begin{array}{l}\text { Globigerinita glutinata } \\
\text { Globigerinoides conglobatus } \\
\text { G. mitra } \\
\text { G. obliquus } \\
\text { G. ruber }\end{array}$ & $\begin{array}{l}1 \\
5\end{array}$ & $\begin{array}{l}5 \\
5\end{array}$ & $\begin{array}{l}2 \\
2\end{array}$ & $\begin{array}{l}1 \\
1\end{array}$ & $\begin{array}{l}1 \\
1\end{array}$ & $\begin{array}{l}1 \\
5 \\
1\end{array}$ & 3 & $\begin{array}{l}1 \\
5\end{array}$ & 5 \\
\hline $\begin{array}{l}\text { G. trilobus } \\
\text { Globoquadrina altispira } \\
\text { G. dehiscens } \\
\text { G. humerosa } \\
\text { G. venezuelana }\end{array}$ & 5 & $\begin{array}{l}4 \\
2\end{array}$ & 4 & $\begin{array}{l}5 \\
1\end{array}$ & & $\begin{array}{l}3 \\
1 \\
1\end{array}$ & 2 & $\begin{array}{l}3 \\
1\end{array}$ & $\begin{array}{l}5 \\
1 \\
1\end{array}$ \\
\hline $\begin{array}{l}\text { Globorotalia acostaensis } \\
\text { G. crassaformis } \\
\text { G. cultrata (dex.) } \\
\text { G. cultrata (sin.) } \\
\text { G. inflata } \\
\end{array}$ & $\begin{array}{l}5 \\
2 \\
1 \\
5\end{array}$ & $\begin{array}{l}5 \\
2 \\
5\end{array}$ & $\begin{array}{l}5 \\
2\end{array}$ & $\begin{array}{l}5 \\
4 \\
1\end{array}$ & & $\begin{array}{l}2 \\
5\end{array}$ & $\begin{array}{l}1 \\
2 \\
2\end{array}$ & $\begin{array}{l}1 \\
4\end{array}$ & $\begin{array}{l}2 \\
5\end{array}$ \\
\hline $\begin{array}{l}\text { G. margaritae } \\
\text { G. plesiotumida } \\
\text { G. scitula } \\
\text { G. tosaensis } \\
\text { Hastigerina siphonifera }\end{array}$ & $\begin{array}{l}5 \\
1 \\
3 \\
\end{array}$ & $\begin{array}{l}5 \\
2\end{array}$ & 1 & 1 & & $\begin{array}{l}1 \\
1\end{array}$ & $\begin{array}{l}1 \\
1 \\
1\end{array}$ & 2 & $\begin{array}{l}1 \\
1\end{array}$ \\
\hline $\begin{array}{l}\text { Orbulina universa } \\
\text { Sphaeroidinellopsis seminulina } \\
\text { S. subdehiscens } \\
\text { Unidentified juveniles and fragments }\end{array}$ & 3 & 3 & 5 & $\begin{array}{l}4 \\
1 \\
5\end{array}$ & $\begin{array}{l}1 \\
5\end{array}$ & $\begin{array}{l}5 \\
1\end{array}$ & 3 & 5 & $\begin{array}{l}5 \\
1\end{array}$ \\
\hline
\end{tabular}

